\title{
Fusarium graminearum Infection and Deoxynivalenol Concentrations During Development of Wheat Spikes
}

\author{
Christina Cowger and Consuelo Arellano
}

First author: United States Department of Agriculture-Agricultural Research Service, Department of Plant Pathology, and second author: Department of Statistics, North Carolina State University, Raleigh 27695. Accepted for publication 5 December 2012.

\section{ABSTRACT}

Cowger, C., and Arellano, C. 2013. Fusarium graminearum infection and deoxynivalenol concentrations during development of wheat spikes. Phytopathology 103:460-471.

Fusarium head blight (FHB) affects whole spikes of small grain plants, yet little is known about how FHB develops following infection, or about the concentration or progression of the mycotoxin deoxynivalenol (DON) in non-grain spike tissues. Fusarium mycotoxin levels in whole smallgrain spikes are of concern to producers of whole-crop silage, as well as users of straw containing chaff for animal bedding or winter livestock rations. A 2-year field experiment was performed in Kinston, NC to reveal the time course of FHB development. Eight winter wheat cultivars with varying levels of FHB resistance were used in the 2006 experiment, and four of them were used in 2007. Plots were spray-inoculated with Fusarium graminearum macroconidia at mid-anthesis. Four durations of post-anthesis mist were applied: $0,10,20$, or 30 days. Spike samples were collected and bulked by plot at $15,25,35,45,55$, and 65 days after anthesis (daa); samples were separated into grain, glume, and rachis fractions. Increasing durations of post-anthesis moisture elevated grain

Fusarium head blight (FHB, or scab) is a damaging disease of wheat (Triticum aestivum) and other small grains worldwide. In the United States, the most important causal agent is Gibberella zeae (anamorph Fusarium graminearum) (22). FHB lowers grain yield and contaminates grain with mycotoxins such as deoxynivalenol (DON). In the United States, the Food and Drug Administration (FDA) has set $1 \mu \mathrm{g} \mathrm{g}^{-1}$ as the recommended ceiling for DON in finished wheat products such as flour, bran, and germ. For feed to be consumed by animals, FDA advisory limits are $5 \mu \mathrm{g} \mathrm{g}^{-1}$ for dairy cattle and swine (for swine, scabby grain must comprise $\leq 20 \%$ of the diet) and $10 \mu \mathrm{g} \mathrm{g}^{-1}$ for beef cattle and chickens (for chickens, scabby grain must comprise $\leq 50 \%$ of the diet).

DON levels in mature wheat grain have been well studied. DON and Fusarium-damaged kernels (FDK) at harvest-ripeness are generally correlated with visual symptoms (31) and are elevated by increasing numbers of wet days following anthesis (6). However, there is relatively less information about how DON concentrations change during the course of grain fill.

\section{Corresponding author: C. Cowger;}

E-mail address: Christina.Cowger@ars.usda.gov

* The $\boldsymbol{e}$-Xtra logo stands for "electronic extra" and indicates that the online version contains one supplemental table.

http://dx.doi.org/10.1094/PHYTO-03-12-0054-R

This article is in the public domain and not copyrightable. It may be freely reprinted with customary crediting of the source. The American Phytopathological Society, 2013.
DON and reduced the effect of cultivar on DON, presumably by affecting the expression of resistance, in all spike tissues. Fusarium-damaged kernels increased from early kernel-hard to harvest-ripe in both years. Percent infected kernels increased from medium-milk to harvest-ripe. During grainfill, DON concentrations declined in grain but increased in rachises and glumes, peaking at early kernel-hard, before declining. Higher mean and maximum DON levels were observed in rachises and glumes than in grain. Estimated whole-spike DON peaked at early kernel-hard. In a highFHB year, whole-plant harvest for forage should be conducted as early as possible. Straw that may be consumed by livestock could contain significant amounts of DON in chaff, and DON can be minimized if straw is sourced from low-symptom crops. Cultivar FHB resistance ratings and disease data should be useful in predicting whole-spike DON levels. Overall, associations between grain DON levels in harvest-ripe and prior samples were stronger the later the prior samples were collected, suggesting limits to the possibility of predicting harvest-ripe grain DON from earlier levels.

Additional keywords: scab.

In the few published reports on DON concentrations and grain infection prior to harvest, various patterns have been observed. In the following examples, disparate results were reported. In field experiments with one winter wheat cultivar in a warm dry year in Ottawa, where spikes were inoculated at anthesis with a conidial suspension, Miller and Young (24) found that DON and ergosterol concentrations in wheat spikes peaked 6 weeks after inoculation, then declined more than threefold by 9 weeks after inoculation. The authors hypothesized that the decline was due to the breakdown of DON by plant enzymes. It was likely on that basis that Teich (38) indicated that DON levels, detectable at $\approx 3$ days after inoculation (dai), peak $\approx 6$ weeks after infection and then begin to decline, reaching a constant level before harvest maturity. In Ontario, Scott et al. (35) sampled five soft winter wheat fields planted to unspecified cultivars with relatively high natural FHB incidences at the three time points of 13 to 20,6 to 13 , and 2 to 9 days before harvest. There was a significant drop in DON from the first to the second sampling, which the authors thought might be due to metabolism by host enzymes. Mean DON levels in this study were quite low $\left(\approx 0.5\right.$ to $3.5 \mu \mathrm{g} \mathrm{g}^{-1}$ at the first date and $\approx 0.09$ to $0.17 \mu \mathrm{g} \mathrm{g}^{-1}$ at harvest). In Braunschweig, Germany, a susceptible winter wheat cultivar was inoculated in the field at midanthesis, and mean DON levels increased steadily from $0 \mathrm{mg} \mathrm{kg}^{-1}$ at 9 dai to $\approx 9 \mathrm{mg} \mathrm{kg}^{-1}$ at 44 to 51 dai, and then declined to slightly $<8 \mathrm{mg} \mathrm{kg}^{-1}$ by 58 dai (20). The researchers speculated that the initial increase in DON concentration was due to precipitation during the first 3 weeks after inoculation. In field plots in Kentucky, Argyris et al. (1) established an intense FHB epidemic using inoculation and irrigation, and found that percent infected 
kernels (PIK) began to rise at $\approx 18$ days after anthesis (daa) and reached levels of 90 to $100 \%$ by 40 daa. In that epidemic, DON levels remained fairly constant from $\approx 28$ to 50 daa although, in the last 10 days, DON declined somewhat in the most susceptible cultivar. Culler et al. (7) sampled spikes of three spring wheat cultivars with varying levels of FHB resistance that were inoculated at anthesis in misted field plots in each of 2 years. They found no clear pattern to grain DON concentrations from early dough, hard dough, kernel hard, and harvest-ripe stages.

Some evidence suggests that very wet conditions may result in low DON levels relative to visible blight symptoms. Lemmens (17) inoculated winter wheat lines from mid-anthesis to 16 daa at two locations in each of 2 years, with one block mist irrigated for 42 daa and the other not irrigated. Visible symptoms and yield reduction were more severe after mist irrigation than in nonirrigated plots but DON was lower; it was unclear whether mist irrigation had produced a smaller initial DON accumulation or had deepened the decline of DON. Some wheat genotypes reacted to mist irrigation with an increase in grain DON content, whereas others responded with a decrease. In their 2-year field experiment, Culler et al. (7) compared 15 to 16 days to 31 to 32 days of postflowering mist, and found that the harvest-ripe grain DON concentration under extended mist irrigation was significantly lower.

DON concentrations in wheat spikes are affected by the timing of infection and colonization. DON appears to play a key role in the spread of $F$. graminearum from the wheat floret into the rachis (11). As $F$. graminearum spreads from an infected spikelet up and down the wheat spike, DON biosynthesis is maximal near the infection front, before symptom development, and diminishes in kernels once they have been colonized (10).

The dynamic nature of DON concentrations in small-grain spikes is likely also partly plant-mediated. Wheat may convert DON to DON-3-glucoside, which is only detected by tandem mass spectrometry (5). As a percentage of DON concentration, DON-3-glucoside concentration was measured at 4 to $12 \%$ in naturally contaminated wheat samples (5) and $14 \%$ in randomly selected hard red spring wheat samples (33). FHB-resistant wheat lines produce a high ratio of DON-3-O-glucoside to DON (18). The quantitative trait locus $Q f h s . n d s u-3 B S$ is closely associated with FHB resistance (40) and has been hypothesized to encode a DON-glucosyl-transferase or its expression regulator (18). Differences in glucosyl-transferases may contribute to the phenotype of more rapidly or profoundly decreasing DON levels during grainfill.

Fusarium spp. infect not only the kernels but also the entire spike of the small-grain plant, and the implications for animal husbandry are, as yet, poorly understood. Wheat or barley straw is used as litter for turkey production (9), and the raising of pigs on straw is gaining in favor (26). Elevated mycotoxin levels in wheat straw used for swine can cause problems because swine consume part of their bedding (e.g., weaner pigs in straw-based systems obtain $\approx 12 \%$ of their total feed intake from straw) (2). Cereal straw and chaff is used as a source of roughage (fiber) in dairy and beef cattle rations, and can provide $\leq 60 \%$ of dry matter feed for wintering beef cows, with chaff comprising $\leq 21 \%$ of the total material and having a higher feed value than stems $(8,9,21)$.

It is known that DON levels are higher in chaff than in kernels of small grains. For example, Miller et al. (25) found that wheat chaff from inoculated field plots of both resistant and susceptible cultivars had $\approx 10$ times the DON of wheat kernels. Rottinghaus et al. (32) found that DON concentrations in chaff from inoculated field plots were poorly correlated with cultivar resistance level, and were approximately four times higher than DON in straw and grain.

Wheat and other small grains are often a partial or primary source of ensiled forage in diets fed to dairy and beef cattle (14). For ensiling of whole-crop cereals, nutrition is maximized by har- vesting plants at the milk to soft-dough stage, or $\approx 250$ to $450 \mathrm{~g}$ $\mathrm{kg}^{-1}$ dry matter $(3,4,12,36)$, although the practical goal of maximizing dry-matter yield may encourage slightly later harvest, at medium dough (37). Whole-crop wheat is sometimes harvested substantially later ( 700 to $800 \mathrm{~g} \mathrm{~kg}^{-1}$ dry matter) and ensiled with alkaline additives to increase digestibility (13). Because DON concentrations are apparently changing in developing grain, they may also be changing from anthesis onward throughout smallgrain spikes, yet nothing is known about these temporal dynamics. Mycotoxins are known to survive the ensiling period and remain a risk to animal health (41).

Thus, DON concentrations appear to be dynamic during grainfill but, to date, preharvest data are inconsistent and available only for grain. A better understanding of the factors that determine harvest-ripe DON levels may help with DON management. To understand FHB development and the progress of DON concentrations in different wheat spike fractions during the grain-fill period, we conducted a field experiment using multiple wheat cultivars with varying resistance levels, as well as different durations of post-anthesis moisture.

\section{MATERIALS AND METHODS}

A 2-year field experiment was conducted to assess the changes in FDK, infected kernels, and DON in three wheat spike fractions (rachis, glumes, and grain) over the course of grain development.

Field experiment location and design. The field experiment was performed in a misted nursery at the Cunningham Research and Extension Center in Kinston, NC. Experimental details were described by Cowger et al. (6); the same plots were used for that study and the presently described one. The same experiment (with different randomizations in the 2 years) was performed in each of two successive field seasons, 2005-06 and 2006-07, hereafter referred to as 2006 and 2007, respectively. In each year, the experiment was planted in a conventionally tilled field (full primary and secondary tillage with burying of crop residues) following corn or soybean.

In the experiment, there was a single section of the field each for misting durations of $0,10,20$, and 30 daa. Due to resource limitations, the durations of misting were present each with a single repetition. Each section consisted of three replicate blocks. Cultivars were randomly assigned to plots within each block, so that each cultivar was subjected to each duration of post-anthesis misting in three replicate plots. Plots were planted with a five-row plot drill, and measured $3.1 \mathrm{~m}$ in length and $1.2 \mathrm{~m}$ in width after trimming ends for evenness of length.

Cultivars for the experiment (Table 1) were chosen as previously described (6) from among regionally adapted lines with similar maturities and a range of putative resistance types (type I, or resistance to primary infection; type II, or resistance to subsequent colonization; or both) (23). For the moderately resistant (MR) cultivars, resistance types were postulated based on incidence, severity, and DON data from the 2000 to 2003 Uniform Southern Soft Red Winter Wheat FHB Screening Nurseries (2730). VA01W-99 was an elite line with pedigree FFR525/93-52-55 (Massey*3/Balkan//Saluda) that had shown a high level of FHB resistance in field experiments (J. Chen and C. Griffey). In the second year, only four of the cultivars used in the first year were included due to effects of a spring freeze on the earliest-maturing cultivars.

Inoculation and misting. Four isolates of $F$. graminearum with proven pathogenicity to wheat were selected to produce inoculum for the field experiment, and were increased individually in aerated 20-liter carboys of Mung bean tea. Mung beans were steeped in boiled, distilled $\mathrm{H}_{2} \mathrm{O}$ at a rate of $40 \mathrm{~g} \mathrm{liter}^{-1}$ for $15 \mathrm{~min}$. The tea was filtered through cheesecloth, autoclaved, and inoculated with macroconidia to provide starter cultures. Carboys containing 12 liters of tea were spiked with $500 \mathrm{ml}$ each of the starter 
cultures, then aerated with sterile air for $\approx 1$ week before centrifugation at 4,000 rpm for $3 \mathrm{~min}$.

A suspension of $10^{5}$ macroconidia $\mathrm{ml}^{-1}$ was used to inoculate field plots. The suspension was composed of equal proportions of spores of the four isolates, and plots were inoculated at midanthesis using a backpack sprayer. Mid-anthesis was considered to be the day on which $50 \%$ of the heads of a given cultivar had extruded anthers; thus, cultivars were inoculated on different dates within a season. The dates of inoculation were 13 to 19 April 2006 and 23 to 25 April 2007.

Roberts 435/436 mist nozzles (Mid-Atlantic Irrigation, Farmville, VA) with 1.4-mm-diameter orifices were mounted on risers at a height of $0.91 \mathrm{~m}$ above the ground and used to provide postanthesis misting. Individual lateral irrigation lines were activated or deactivated to furnish the different durations of post-anthesis mist. Misting occurred for $2 \mathrm{~min}$ during each 20-min period for $3 \mathrm{~h}$ each in the morning and afternoon (8:00 to 11:00 a.m. and 2:00 to 5:00 p.m.), for a total of $36 \mathrm{~min}$ per day, using a programmable timer. Actual flow delivered was $0.275 \mathrm{~mm} \mathrm{~min}{ }^{-1}$, taking sprinkler overlap into account. Post-anthesis mist periods $(0,10,20$, or 30 daa) started during the inoculation period (17 April 2006 and 23 April 2007).

Buffer plots could not be planted between all treatments due to limited misted nursery space. However, buffer strips of the MR 'NC-Neuse' were planted to avoid interference of irrigation treatments, and borders of NC-Neuse were planted around the experiment.

Disease assessment and sample collection. The assessment of disease severity was conducted on each cultivar 20 days after it had been inoculated. Forty heads were chosen blindly in each plot, avoiding outside rows and terminal $0.3-\mathrm{m}$ portions of plots. Severity was assessed as the percentage of diseased spikelets in the 40 heads (diseased spikelets/total spikelets $\times 100$ ).

To assay kernel symptoms and DON, 30 spikes were blindly harvested from each plot and bulked by plot on each of six dates $\approx 10$ days apart, from milk stage to $\approx 20$ days after harvest-ripeness. The collections were made $15,25,35,45,55$, and 65 daa, with the first collection from all plots on a single day 15 days after the mid-point of the 6-day interval between the earliest and latest inoculation dates in 2006, the second collection 10 days later, and so on. In 2007, there was a 2-day interval between the earliest and latest inoculation dates; samples were collected at $\approx 10$-day intervals starting 15 days from the mid-point of that 2-day interval. The outer edges and $0.5 \mathrm{~m}$ at the ends of each plot were avoided to minimize edge effects. From each plot, spike samples were manually separated into grain, glume, and rachis fractions. In 2006, the grain fraction was weighed; in 2007, all fractions were weighed.

To determine the percentage of FDK, 100 kernels were selected arbitrarily from the sample for each plot and visually inspected for gray-white or pink discoloration and mycelial growth. Grain was assayed for DON concentration by enzyme-linked immunosorbent assay using EZ-Tox DON Kits (Diagnostix, Mississauga, $\mathrm{ON}$, Canada), with detection limits of 0.5 to $5 \mu \mathrm{g} \mathrm{g}^{-1}$; for results exceeding $5 \mu \mathrm{g} \mathrm{g}^{-1}$, samples were diluted and reanalyzed appropriately.

The PIK in the 2006 and 2007 samples was determined by surface sterilizing 25 arbitrarily chosen kernels per sample for 2 min in $5 \%$ bleach, and plating the kernels on Komada's selective medium (15). Kernels were incubated 7 days in a growth chamber at $22^{\circ} \mathrm{C}$ on a 12 -h light-and-dark cycle. Identification of infected kernels was based on fluffy peach-white mycelial growth and characteristic discoloration of the medium beneath the kernels. Spore morphology was determined microscopically where necessary to confirm infection by $F$. graminearum.

Statistical analysis. In each year, the experiment followed a nested or hierarchical design, with duration of post-anthesis moisture (mist duration) as a main-unit (single-repetition) factor; subunits (blocks) nested within each mist-duration mainunit, three repetitions for each; and cultivar as a sub-subunit factor, with all cultivars represented within each block. Sample date was a repeated-measures fixed-effect factor, with a number of samples taken from each plot. Mist duration, cultivar, and sample were crossed fixed factors. Data were analyzed following a linear mixed model with mist duration, cultivar, sample dates, and their interactions as fixed effects, and block nested within mist duration and interactions involving block as random effects. Sample dates were considered repeated measures and their variability was modeled as a heterogeneous compound symmetry, with the subject identified by the combination mist durationblock-cultivar, where six repeated measures were taken at 10-day intervals. This covariance structure estimated a separate residual variance for each sample date and common compound symmetry coefficient.

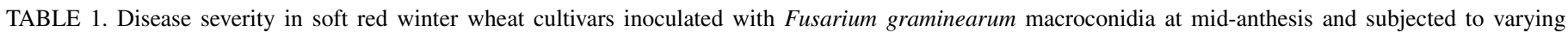
durations of post-anthesis mist in a field nursery in Kinston, NC

\begin{tabular}{|c|c|c|c|c|c|c|c|}
\hline \multirow[b]{2}{*}{ Cultivar } & \multirow[b]{2}{*}{ Number $^{\mathrm{x}}$} & \multirow[b]{2}{*}{ Date $^{y}$} & \multicolumn{5}{|c|}{ Severity $(\%)$ at each mist duration (days after anthesis) ${ }^{\mathrm{z}}$} \\
\hline & & & 0 & 10 & 20 & 30 & Mean \\
\hline \multicolumn{8}{|l|}{2006} \\
\hline Ernie & 9600360 & 14 April & 1.0 & 1.9 & 6.4 & 8.5 & $4.5 \mathrm{a}$ \\
\hline VA01W99 & Experimental & 13 April & 2.4 & 8.2 & 14.3 & 10.3 & $8.8 \mathrm{ab}$ \\
\hline NC-Neuse & 200400303 & 19 April & 1.3 & 8.8 & 15.1 & 11.1 & $9.1 \mathrm{ab}$ \\
\hline V-Tribute & 632689 & 15 April & 2.9 & 9.0 & 14.0 & 18.0 & $11.0 \mathrm{~b}$ \\
\hline C9474 & 9400188 & 19 April & 1.3 & 16.1 & 17.7 & 16.1 & $12.8 \mathrm{~b}$ \\
\hline USG 3650 & W711 & 19 April & 3.2 & 14.5 & 26.3 & 14.1 & $14.5 \mathrm{bc}$ \\
\hline C9184 & 200200135 & 17 April & 11.0 & 27.1 & 34.9 & 41.8 & $28.7 \mathrm{c}$ \\
\hline USG 3592 & 200400110 & 15 April & 5.7 & 23.2 & 59.8 & 50.4 & $34.8 \mathrm{c}$ \\
\hline Mean & $\ldots$ & $\ldots$ & $3.6 \mathrm{a}$ & $13.6 \mathrm{ab}$ & $23.6 \mathrm{~b}$ & $21.3 \mathrm{~b}$ & $\ldots$ \\
\hline \multicolumn{8}{|l|}{2007} \\
\hline NC-Neuse & $\ldots$ & 25 April & $1.2 \mathrm{a}$ & $4.9 \mathrm{a}$ & $9.6 \mathrm{a}$ & $5.0 \mathrm{a}$ & 5.2 \\
\hline C9474 & $\ldots$ & 23 April & $0.2 \mathrm{a}$ & $7.3 \mathrm{a}$ & $8.8 \mathrm{a}$ & $7.5 \mathrm{ab}$ & 6.0 \\
\hline USG 3650 & $\ldots$ & 23 April & $0.9 \mathrm{a}$ & $15.5 \mathrm{~b}$ & $40.0 \mathrm{~b}$ & $6.6 \mathrm{ab}$ & 15.8 \\
\hline C9184 & $\ldots$ & 25 April & $4.3 \mathrm{a}$ & $92.4 \mathrm{c}$ & $90.2 \mathrm{c}$ & $16.7 \mathrm{~b}$ & 50.9 \\
\hline Mean & $\ldots$ & $\ldots$ & 1.6 & 30.0 & 37.1 & 9.0 & $\ldots$ \\
\hline
\end{tabular}

${ }^{x}$ Plant introduction or Plant Variety Protection tagging number.

y Inoculation date. Each cultivar was inoculated at 50\% anthesis.

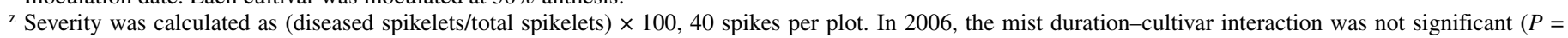
0.214). In 2007, the interaction was significant $(P<0.0001)$; simple-effects tests indicated that cultivar differences were significant at every mist duration but mist duration differences were only significant for USG 3650 and C9184. Within a year-column or year-row combination, means followed by the same letter are not significantly different $(P \leq 0.05)$. 
Due to the small number of years (two) and environmental differences between the years, each year was analyzed separately. A combined analysis, with year treated as a fixed-effect factor, showed a nonsignificant effect for mist duration when tested against the year-mist duration interaction, with $P$ values for DON of 0.10 (grain), 0.10 (glumes), and 0.9 (rachis). Significant ( $\alpha=$ 0.05 ) interaction effects were year-sample date, year-mist duration-sample date, and mist duration-cultivar (year) for all three measures of DON (grain, glumes and rachis). Within each year, no test for the mist-duration main effect was considered.

To determine the effects of the independent experimental factors on grain weight, PIK, FDK, and DON, a general linear mixed model was fitted to the data using the MIXED procedure in SAS (version 9.1.3; SAS Institute, Cary, NC). Inspection of residual plots indicated the need for square-root transformation of FDK and DON data to attain normality and homogeneity of variances. The restricted maximum likelihood method was used to calculate variance components in the mixed model. Fixed effects were estimated by solving the mixed-model equations. The Kenward-Roger correction was used to reduce bias in the estimated covariance matrix of fixed and random effects and apply a Satterthwaite-type adjustment in the calculation of denominator degrees of freedom for the approximated $F$ statistic used in testing the fixed effects. Least-squares means separation was performed using Tukey's test and the pdmix800.sas macro (34).

The statistical model was given by $y_{i j k l}=\mu+\alpha_{i}+b_{i j}+\gamma_{k}+$ $(\alpha \gamma)_{i k}+g_{i j k}+\omega_{l}+(\alpha \omega)_{i l}+(\gamma \omega)_{k l}+(\alpha \gamma \omega)_{i k l}+e_{i j k l}$, where $\alpha_{i}$ is the fixed effect for mist duration, $i=1,2,3,4 ; b_{i j}$ is the random effect of the $j$ th block within the $i$ th level of mist duration, $j=1,2,3, b_{i j} \sim$ $N\left(0, \sigma_{b}^{2}\right) ; \gamma_{k}$ is the fixed effect of cultivar, $k=1,2,3, \ldots, K(K=8$ for 2006 and $K=4$ for 2007); $(\alpha \gamma)_{i k}$ is the fixed interaction effect between mist duration and cultivar for the $i$ th mist duration and $k$ th cultivar; $g_{i j k}$ is the random effect associated with the cultivarblock (mist duration) interaction, for the $k$ th cultivar, $j$ th block, and $i$ th mist duration, $g_{i j k} \sim N\left(0, \sigma_{b}^{2}\right)$; $\omega_{l}$ is the fixed effect for sample date, $l=1,2, . .6 ;(\alpha \omega)_{i l}$ is the fixed interaction effect between mist duration and sample date, for the $i$ th mist duration and $l$ th sample date; $(\gamma \omega)_{k l}$ is the fixed interaction effect between cultivar and sample date, for the $k$ th cultivar and $l$ th sample date; $(\alpha \gamma \omega)_{i k l}$ is the fixed interaction effect between mist duration, cultivar, and sample date, for the $i$ th mist duration, $k$ th cultivar, and $l$ th sample date; and $e_{i j k l}$ is the random effect associated with the $l$ th sample date for the observational unit identified by the $k$ th cultivar, $j$ th block, and $i$ th mist duration, $e_{i j k l} \sim N\left(0, \sigma_{b}^{2}\right)$.

When necessary, an alternative model was used that took into account the heterogeneity of the random effects, and a separate residual variance was estimated for each sample date, as follows: $y_{i j k l}=\mu+\alpha_{i}+b_{i j}+\gamma_{k}+(\alpha \gamma)_{i k}+\omega_{l}+(\alpha \omega)_{i l}+(\gamma \omega)_{k l}+(\alpha \gamma \omega)_{i k l}+$ $e_{i j k l}$.

The corresponding assumption was that, for each observational unit, $e_{i j k l} \sim N(0, R)$, where $R$, the covariance matrix between the set of six sample date measurements, was represented by

$$
R=\left[\begin{array}{cccccc}
\sigma_{1}^{2} & & & & & \\
\sigma_{1} \sigma_{2} \rho & \sigma_{2}^{2} & & & & \\
\sigma_{3} \sigma_{1} \rho & \sigma_{3} \sigma_{2} \rho & \sigma_{3}^{2} & & & \\
\sigma_{4} \sigma_{1} \rho & \sigma_{4} \sigma_{2} \rho & \sigma_{4}^{2} \sigma_{3}^{2} \rho & \sigma_{4}^{2} & & \\
\sigma_{5} \sigma_{1} \rho & \sigma_{5} \sigma_{2} \rho & \sigma_{5} \sigma_{3} \rho & \sigma_{5} \sigma_{4} \rho & \sigma_{5}^{2} & \\
\sigma_{6} \sigma_{1} \rho & \sigma_{6} \sigma_{2} \rho & \sigma_{6} \sigma_{3} \rho & \sigma_{6} \sigma_{4} \rho & \sigma_{6} \sigma_{5} \rho & \sigma_{6}^{2}
\end{array}\right]
$$

with $\rho$ representing the common correlation between any two measurements and $\sigma_{l}^{2}$ the variance associated with measurements taken at the $l$ th sample date. The Kenward-Roger correction was used to reduce bias in the estimated covariance matrix of fixed and random effects, and a Satterthwaite-type adjustment was applied in the calculation of denominator degrees of freedom for the approximated $F$ statistic used in testing the fixed effects $(19,39)$. Covariance parameter estimates for FDK, PIK, and DON are given in Supplemental Table 1.

An analysis was conducted to determine potential association of DON levels of each spike fraction (grain, glumes, and rachis) with DON levels in the other spike fractions and with index, FDK, and PIK, after accounting for the effect of experimental factors. This was done by calculating partial correlation coefficients using the multivariate analysis of variance procedure in SAS.

A covariance analysis was also performed to evaluate the relationships of DON levels at sample 5 (harvest-ripeness) with DON levels at each of the prior sampling points. The purpose was to explore whether determination of grain DON prior to harvest could be useful in forecasting DON levels at harvest. The analysis was performed on square-root-transformed DON in the 2006 data set only, because it was larger than the 2007 dataset and allowed for better estimation of the fixed and random effects. For this analysis, mist duration was considered a fixed-effect factor. The model fitted included a separate intercept and slope for each mist duration level. Cultivar was treated as a random-effect factor, because the tested cultivars possessed diverse resistance phenotypes and the question of interest was the relationship of harvestripe DON to DON at earlier stages, using a range of cultivar responses. The standard error for calculated slopes (regression coefficients) included the variability associated with cultivar effects on square-root-transformed DON. For each level of mist duration, the statistical model was $y_{i j k}=\mu+\alpha_{i}+b_{i j}+g_{k}+(\alpha g)_{i k}+$ $\beta_{i l}+x_{i j k}^{1}+e_{i j k}$, where $\alpha_{i}$ was the fixed effect for mist duration, $i=$ $1,2,3,4 ; b_{i j}$ was the random effect of the $j$ th block within the $i t h$ level of mist duration, $j=1,2,3, b_{i j} \sim N\left(0, \sigma_{b}^{2}\right) ; g_{k}$ was the random effect of cultivar, $k=1,2,3, \ldots 8, g_{k} \sim N\left(0, \sigma_{g}^{2}\right) ;(\alpha g)_{i k}$ was the random effect of the interaction of mist duration and cultivar, $(\alpha g)_{i k} \sim N\left(0, \sigma_{\alpha g}{ }^{2}\right) ; \beta_{i l}$ was the regression coefficient for the covariable $X^{1}=\operatorname{sqrt}(\mathrm{DON})$ at $l$ th sample date, in the $i$ th level of mist duration, $l=1,2,3,4,5,6$; and $e_{i j k}$ represented the uncontrolled random effects associated with the observation identified by the $i t h$ mist duration, $j$ th repetition, and $k$ th cultivar, $e_{i j k} \sim N\left(0, \sigma_{g}^{2}\right)$.

The model estimated a separate intercept and slope for each mist duration level. The hypotheses tested were (i) $\mathrm{H}_{\mathrm{o}}$ : no mist duration effect (i.e., a common intercept was enough), $\mathrm{H}_{\mathrm{o}}: \alpha_{i}=0$, $i=1, \ldots 4$; and (ii) $\mathrm{H}_{\mathrm{o}}$ : slopes for all mist durations equaled $0, \mathrm{H}_{\mathrm{o}}$ : $b_{i l}=0, i=1, \ldots 4$.

Total DON amounts in wheat spikes were estimated for the different sample dates as follows. Weights for the three spike fractions (glumes, grain, and rachises) were available for 2007 but not for 2006. The weights of the spike fractions were averaged across cultivars and misting treatments for each sample date, and the relative proportions of fraction weights on each date in 2007 were used for estimates in both years, on the assumption that the physiological process of spike development is relatively invariant. Total DON per spike was then estimated by weighting the concentration of DON in each spike fraction by the proportion of total spike weight contributed by that fraction, and summing the results.

\section{RESULTS}

The spring 2006 season was more conducive to FHB than the spring 2007 season, primarily due to more frequent rains in the 10- to 20-mm-per-day range in the post-anthesis period (Fig. 1). The period of anthesis and grain development was considerably drier in 2007 than in 2006: altogether, between mid-anthesis and the 55-daa sample date (harvest-ripeness), there was $166 \mathrm{~mm}$ of rain in 2006 and $94 \mathrm{~mm}$ of rain in 2007.

In the analysis of FDK, PIK, and DON, the three measures of grain disease, year interacted significantly with sample date in each case. Therefore, data are presented for the 2 years separately (Table 2). 
Severity. Assessments of visual symptoms were conducted at approximately the point at which the 20-daa mist treatment finished (Table 1). Thus, the values for the 30-daa mist treatment were not expected to differ significantly from those for the 20-daa treatment.

Mist duration and cultivar did not interact significantly in 2006 $(P=0.214)$. Mist duration and cultivar each had a significant effect on severity $(P \leq 0.0004)$. As expected, the two cultivars chosen for their susceptibility ('C9184' and 'USG 3592') had higher levels of severity, although they were not significantly different from 'USG 3650'.

In 2007 , the interaction was significant $(P<0.0001)$; a simpleeffects test indicated that cultivar differences were significant for each duration of mist applied but mist duration differences were only significant for USG 3650 and C9184. The low severities for USG 3650 and C9184 under the 30-daa mist treatment may have been due to patchy occurrence of Wheat soilborne mosaic virus (WSBMV).

Weight. In both years, mean 30-spike grain weights increased to a maximum in the third sample, collected at early kernel hard stage (Fig. 2A and B). After that, grain weights declined slightly, as was expected as the crop dried down. In both years, grain weights were significantly related to cultivar and mist duration $(P \leq 0.0003)$

Proportions of the weights of the various spike fractions, averaged across cultivars and misting treatments for 2007, are shown in Figure 3. The weight of the grain fraction relative to those of glumes and spikes increased until the 35-daa sample, when it leveled out for the remainder of the season.

Kernel damage. Percent FDK was assessed for the 35- through 65-daa samples in both years (Fig. 2C and D), the kernels in the previous samples being too small to accurately detect differences by visual inspection. Sample date interacted significantly with mist duration in both years $(P \leq 0.007)$. Misting had a strong elevating effect on FDK in $2006(P \leq 0.0001$ at all sample dates, simple-effects test) but less so in 2007 ( $P \geq 0.071$ for all samples except 55 daa, $P=0.014$, simple-effects test) (Fig. 2C and D). Post-anthesis mist tended to elevate FDK in the 45- and 55-daa samples, and lack of post-anthesis mist was associated with the lowest level of FDK at all sampling points.

Sample date interacted significantly with cultivar in 2006 but not in 2007 (Table 2). Cultivars differed significantly for FDK at all sample dates in both years ( $P \leq 0.033$, simple-effects test).

In both years, sample was a significant source of variation, and percent FDK increased and peaked after the 35-daa sample (Table 2; Fig. 2C and D). Sample date was a larger source of variation in FDK in 2006 than in 2007, particularly due to the 30-daa mist treatment in 2006. FDK declined in the 65-daa sample, although more so in 2006 than in 2007.

PIK. The PIK was assayed for all six samples in 2006 and for all but the 15-daa sample in 2007 (Fig. 2E and F). Averaging across all independent variables, mean PIK was higher in 2006, the wetter year, than in 2007 (Fig. 2E and F), and it was higher at 55 daa (harvest-ripeness) in 2006 than in 2007 (63 versus 28\%, $P<0.05$ ).

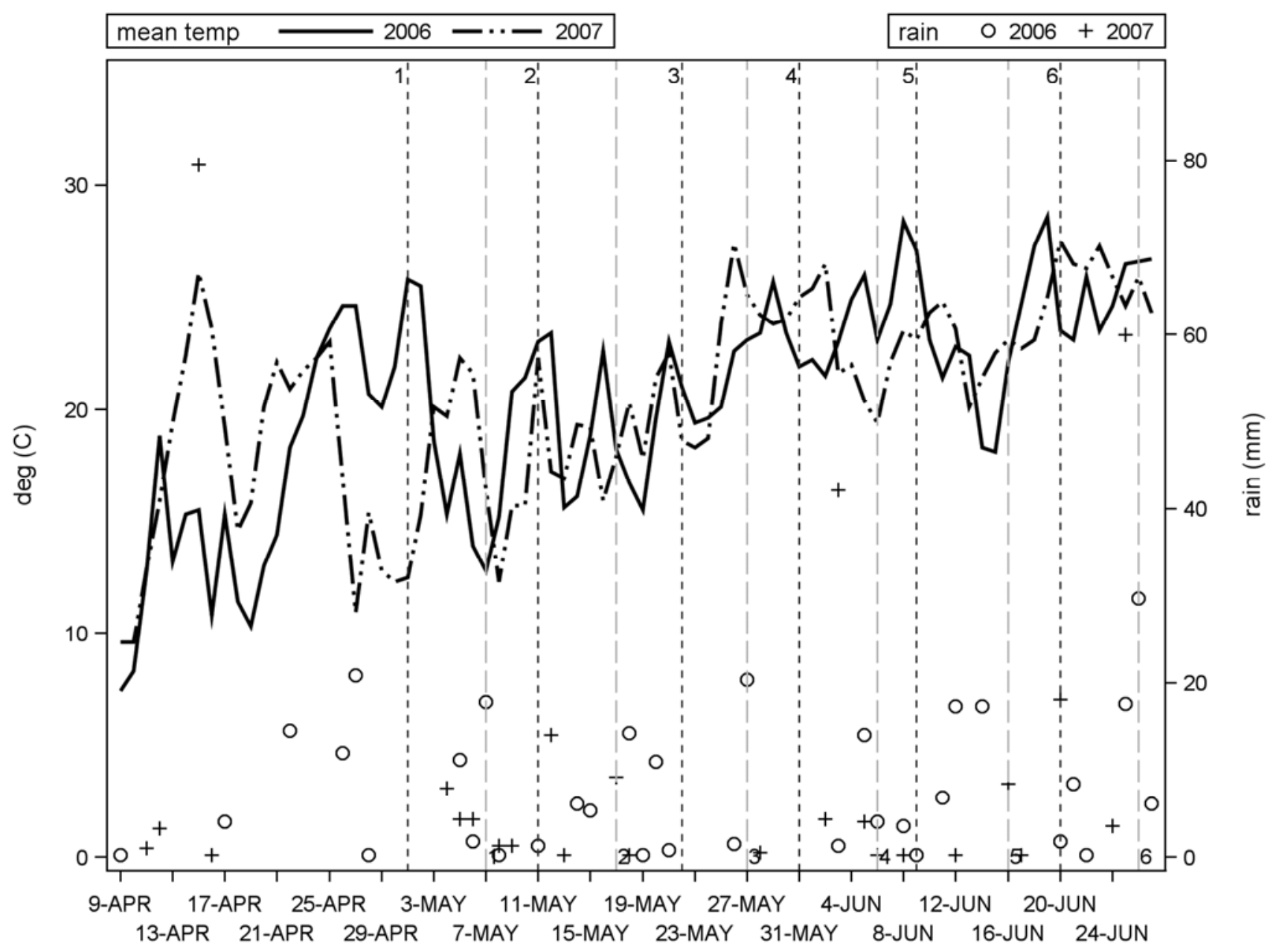

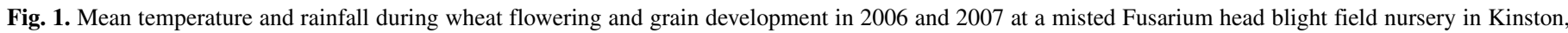

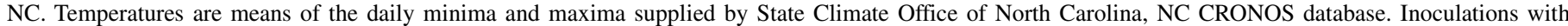

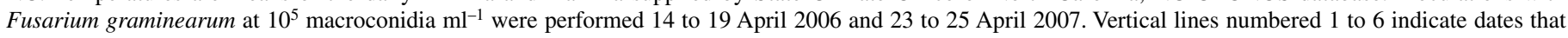
wheat spike samples were collected (short dashes $=2006$, long dashes $=2007$ ). 
Sample date interacted significantly with mist duration in both years (Table $2, P \leq 0.034$ ). Misting increased PIK at all sample dates in $2006(P \leq 0.004)$, and at 35, 55, and 65 daa in 2007 (simple-effects test, $P \leq 0.037 ; P \geq 0.243$ for 25 and 45 daa). That is, as with FDK, mist duration affected PIK in both years but the effect was greater in 2006 than in 2007 (Table 2; Fig. 2C and D).
Sample date interacted significantly with cultivar in both years $(P \leq 0.006$, Table 2$)$. Cultivars differed significantly for PIK at all sample dates in $2006(P \leq 0.026)$ and at all dates in 2007 except 35 daa $(P \leq 0.069)$. Averaged across mist durations, the two most susceptible cultivars, USG 3592 and C9184, had the highest or second-highest PIK at all of the significantly differing sample dates prior to 65 daa in both years.

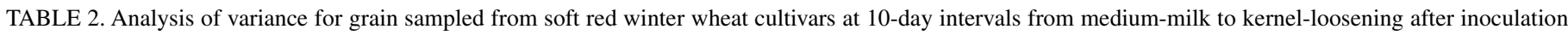
at mid-anthesis with Fusarium graminearum macroconidia in Kinston, NC, in 2006 and 2007 y

\begin{tabular}{|c|c|c|c|c|c|c|c|c|c|}
\hline \multirow[b]{2}{*}{ Year, variation $^{\mathrm{z}}$} & \multicolumn{3}{|c|}{ Fusarium-damaged kernels } & \multicolumn{3}{|c|}{ Percent infected kernels } & \multicolumn{3}{|c|}{ Deoxynivalenol } \\
\hline & df & $F$ value & $P$ value & df & $F$ value & $P$ value & df & $F$ value & $P$ value \\
\hline \multicolumn{10}{|l|}{2006} \\
\hline Mist & $3, \mathrm{n} / \mathrm{a}$ & $\ldots$ & $\ldots$ & $3, \mathrm{n} / \mathrm{a}$ & $\ldots$ & $\ldots$ & $3, \mathrm{n} / \mathrm{a}$ & $\ldots$ & $\ldots$ \\
\hline Cult & 7,222 & 13.51 & $<0.0001$ & $7,53.3$ & 13.48 & $<0.0001$ & $7,55.7$ & 10.51 & $<0.0001$ \\
\hline Mist $\times$ Cult & 21,222 & 1.77 & 0.0232 & $21,52.2$ & 1.75 & 0.0525 & $21,55.3$ & 1.64 & 0.0721 \\
\hline Date & 3,223 & 66.33 & $<0.0001$ & 5,139 & 222.57 & $<0.0001$ & 5,142 & 83.32 & $<0.0001$ \\
\hline Mist $\times$ Date & 9,223 & 3.38 & 0.0007 & 15,193 & 11.38 & $<0.0001$ & 15,195 & 16.12 & $<0.0001$ \\
\hline Cult $\times$ Date & 21,222 & 3.29 & $<0.0001$ & 35,217 & 1.79 & 0.0064 & 35,220 & 1.70 & 0.0121 \\
\hline Mist $\times$ Cult $\times$ Date & 63,222 & 1.48 & 0.0209 & 105,202 & 0.75 & 0.9471 & 105,205 & 1.11 & 0.2691 \\
\hline \multicolumn{10}{|l|}{2007} \\
\hline Mist & $3, \mathrm{n} / \mathrm{a}$ & $\ldots$ & $\ldots$ & $(3, \mathrm{n} / \mathrm{a})$ & $\ldots$ & $\ldots$ & $3, \mathrm{n} / \mathrm{a}$ & $\ldots$ & $\ldots$ \\
\hline Cult & 3,118 & 80.24 & $<0.0001$ & $3,26.4$ & 22.73 & $<0.0001$ & $3,26.4$ & 44.18 & $<0.0001$ \\
\hline Mist $\times$ Cult & 9,118 & 4.56 & $<0.0001$ & $9,26.3$ & 1.50 & 0.2008 & $9,26.3$ & 1.47 & 0.2044 \\
\hline Date & 3,118 & 8.57 & $<0.0001$ & 4, 67.3 & 99.60 & $<0.0001$ & $4,67.3$ & 6.59 & $<0.0001$ \\
\hline Mist $\times$ Date & 9,118 & 2.70 & 0.0067 & $12,86.3$ & 2.00 & 0.0338 & $12,86.3$ & 2.86 & 0.0009 \\
\hline Cult $\times$ Date & 9,118 & 1.47 & 0.1679 & $12,86.3$ & 6.08 & $<0.0001$ & $12,86.3$ & 2.16 & 0.0126 \\
\hline Mist $\times$ Cult $\times$ Date & 27,118 & 2.46 & 0.0005 & 36,90 & 1.06 & 0.3972 & 36,90 & 1.10 & 0.3389 \\
\hline
\end{tabular}

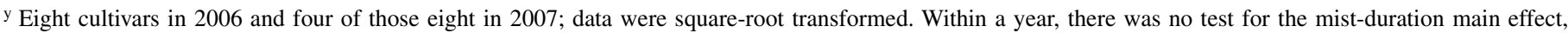
which was the main unit with a single repetition. $\mathrm{df}=$ degrees of freedom: numerator, denominator; $\mathrm{n} / \mathrm{a}=\mathrm{not}$ applicable. Covariance parameter estimates appear in Supplemental Table 1.

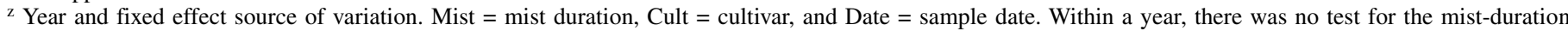
main effect, which was the main unit with a single repetition.

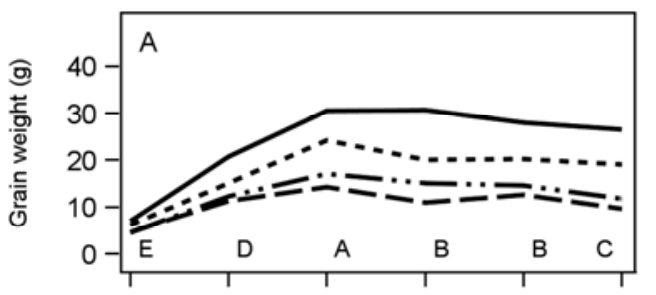

15 daa 25 daa 35 daa 45 daa 55 daa 65 daa

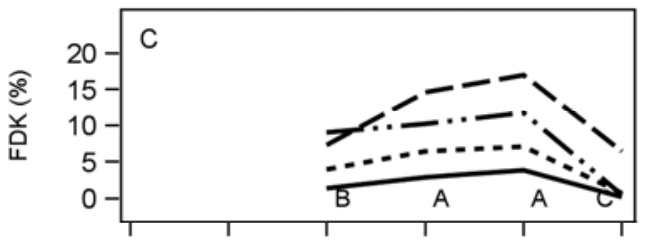

15 daa 25 daa 35 daa 45 daa 55 daa 65 daa

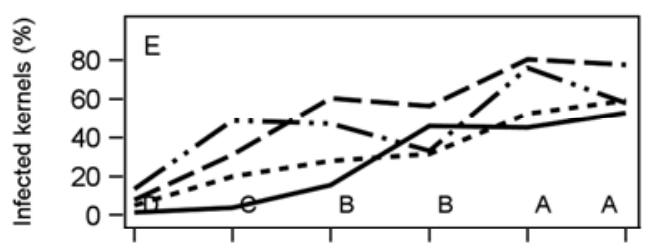

15 daa 25 daa 35 daa 45 daa 55 daa 65 daa

Spike sample

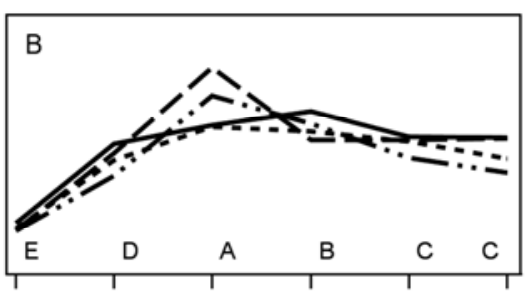

15 daa 25 daa 35 daa 45 daa 55 daa 65 daa

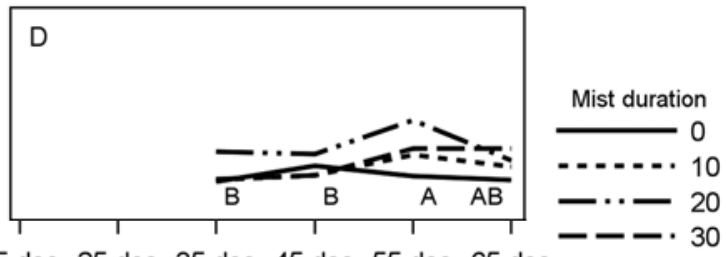

15 daa 25 daa 35 daa 45 daa 55 daa 65 daa

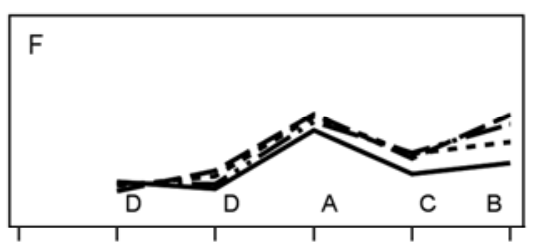

15 daa 25 daa 35 daa 45 daa 55 daa 65 daa

Spike sample

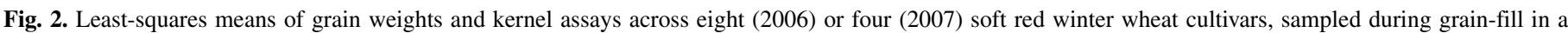

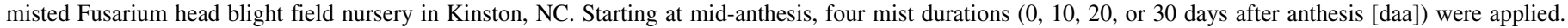

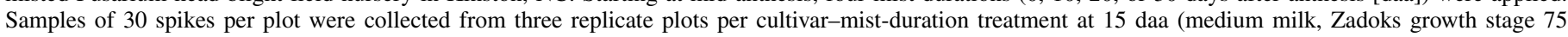

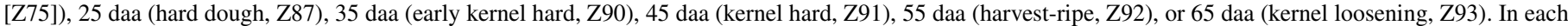

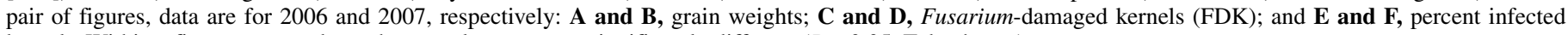
kernels. Within a figure, means above the same letter are not significantly different $(P \leq 0.05$, Tukey's test $)$. 
The largest source of variation in PIK was sample date (Table 2). PIK generally increased on successive sampling dates in 2006 through 55 daa (harvest-ripeness); averaging across cultivars and mist durations, means increased significantly on dates 2,3 , and 5 (Fig. 2E). In 2007, PIK was higher in the later samples than in the earlier ones (Fig. 2D). Values for the 45-daa sample in 2007 were high (Fig. 2F), possibly related to the 42-mm rain event on 3 June (Fig. 1), 4 days before collection of the 45-daa sample.

DON. Averaging across cultivars, mean DON reached higher maximum concentrations in rachis and glume tissues than in grain for all mist durations in both years (Figs. 4 and 5). Whereas DON concentrations generally declined in grain from 15 or 25 daa

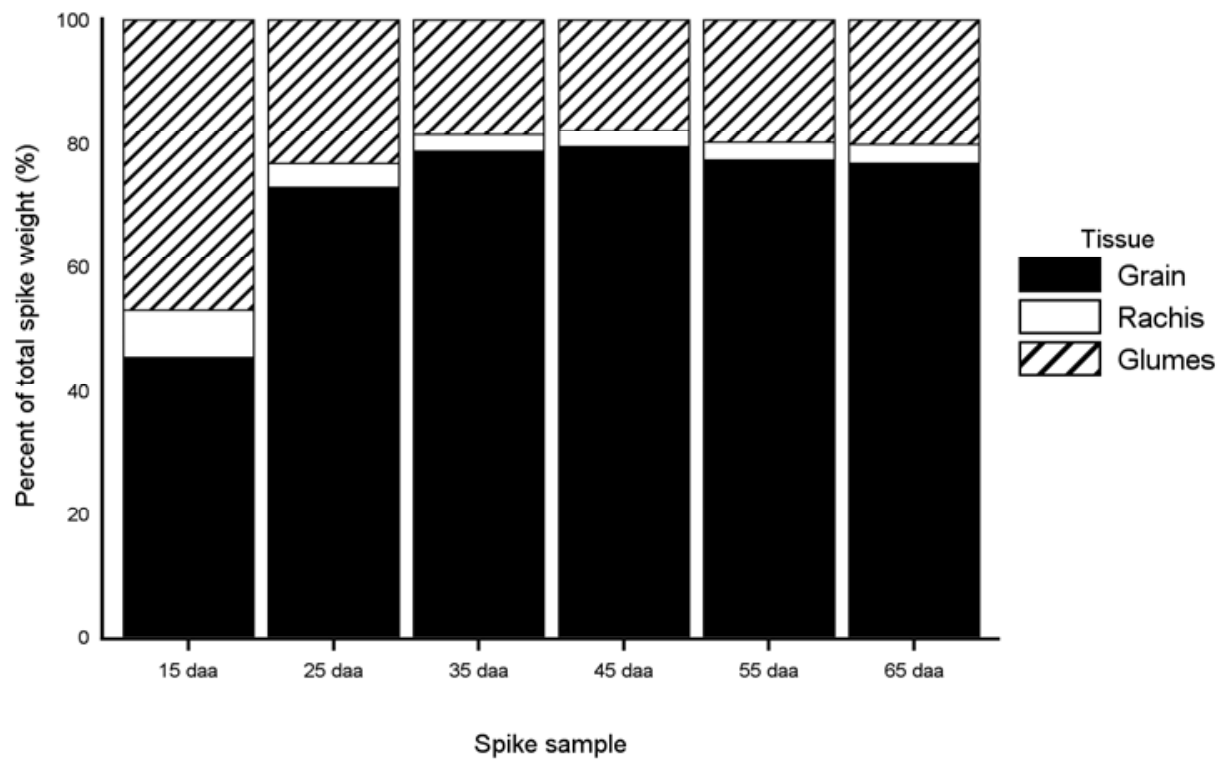

Fig. 3. Spike fractions by weight. Data averaged across four soft red winter wheat cultivars inoculated with Fusarium graminearum macroconidia at mid-anthesis, subjected to four post-anthesis mist durations, and sampled during grain-fill in a misted Fusarium head blight field nursery in Kinston, NC, in 2007.
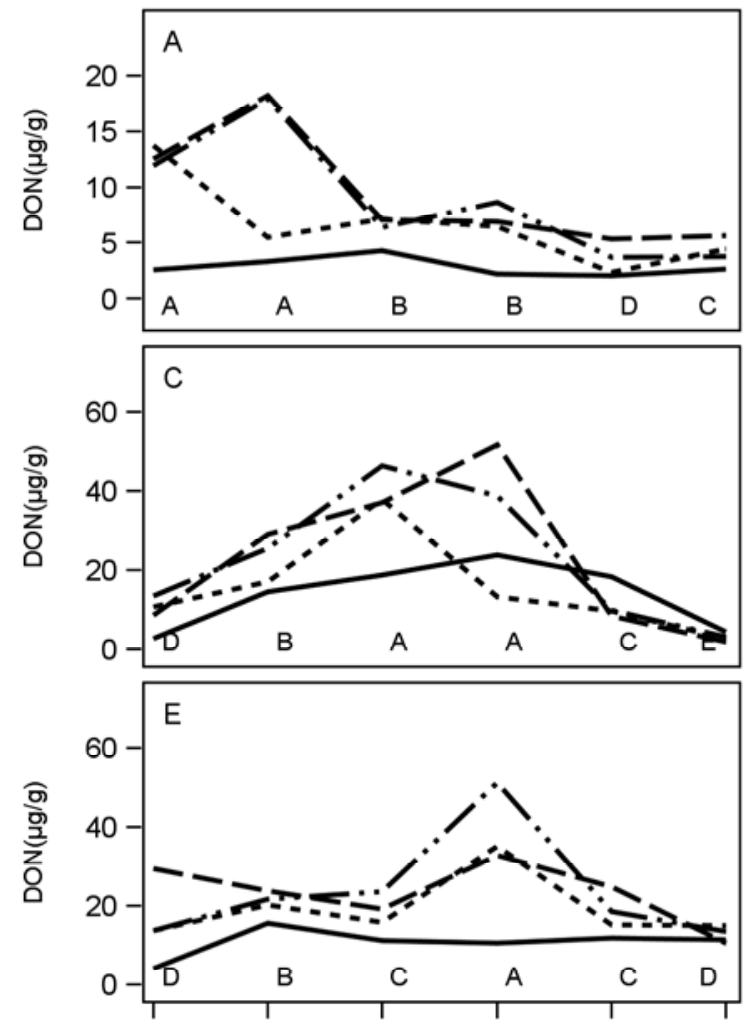

15 daa 25 daa 35 daa 45 daa 55 daa 65 daa
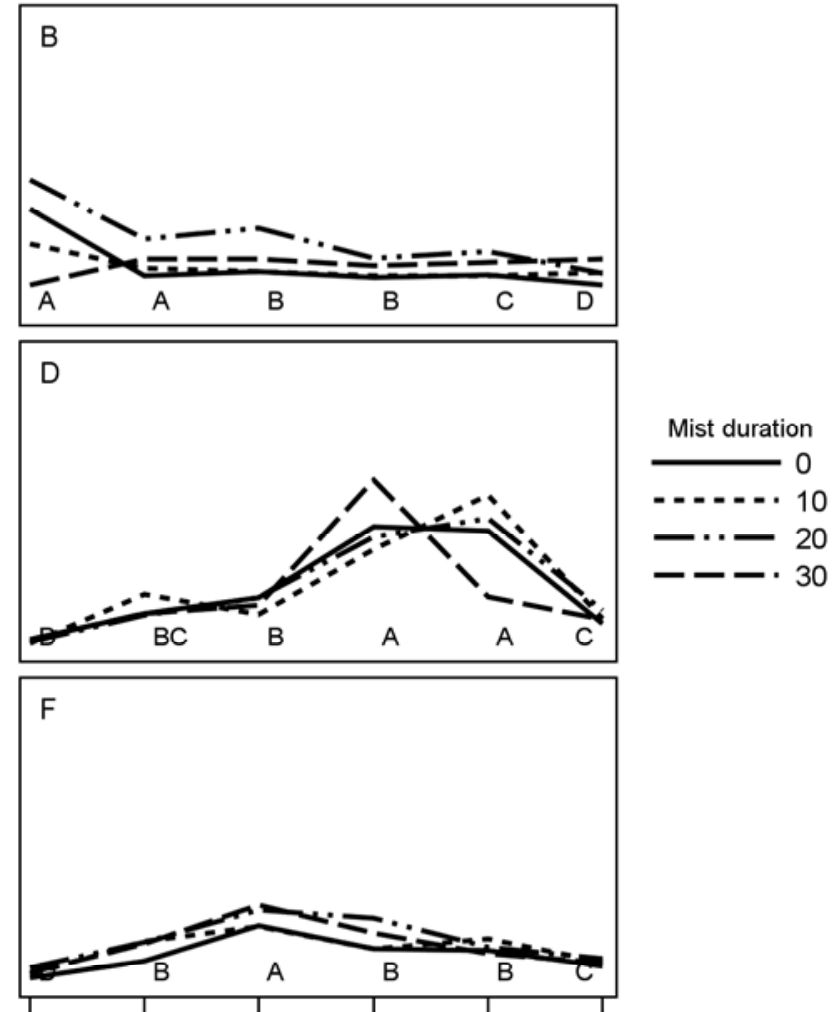

15 daa 25 daa 35 daa 45 daa 55 daa 65 daa

Fig. 4. Least-squares means of deoxynivalenol (DON) in different spike fractions by misting treatment averaged across eight (2006) or four (2007) soft red winter wheat cultivars, sampled during grain-fill in a misted Fusarium head blight field nursery in Kinston, NC. Starting at mid-anthesis, four mist durations $(0,10$, 20, or 30 days after anthesis [daa]) were applied. Samples of 30 spikes per plot were collected from three replicate plots per cultivar-mist-duration treatment at 15 daa (medium milk, Zadoks growth stage 75 [Z75]), 25 daa (hard dough, Z87), 35 daa (early kernel hard, Z90), 45 daa (kernel hard, Z91), 55 daa (harvest-ripe, Z92), or 65 daa (kernel loosening, Z93). In each pair of figures, data are for DON concentrations in 2006 and 2007, respectively: A and B, grain; C and D, rachis; and E and $\mathbf{F}$, glumes. Within a figure, means above the same letter are not significantly different $(P \leq 0.05$, Tukey's test). 
onward, DON levels generally reached their peak in the other spike tissues later (on average, at 45 daa).

For all spike fractions, increasing durations of mist tended to reduce the effect of cultivar on DON in both years (Table 3). In other words, increasing numbers of post-anthesis wet days changed cultivar DON resistance rankings in all types of spike tissues. This trend was greater in 2006, the year in which mist duration tended to affect measures of disease, than in 2007, when it did not.

Grain. When averaged across samples, cultivars, and mist treatments, mean grain DON was nearly twice as high in 2006 as in 2007 (7.2 and $3.8 \mu \mathrm{g} / \mathrm{g}$, respectively) and, at harvest ripeness, it was 3.2 versus $2.3 \mu \mathrm{g} \mathrm{g} \mathrm{g}^{-1}$. Averaging across cultivars and mist levels, grain DON levels were higher earlier in grain-fill and declined toward harvest in both years (Fig. 4A and B). Sample date was by far the largest source of variation in grain DON in 2006 and was significant in 2007 although, in the latter year, cultivar accounted for a larger share of the variation (Table 2). When the data from both years were combined, sample date interacted significantly with year, cultivar, and mist duration $(P \leq$ 0.0009).

Mist duration interacted significantly with sample in both years $(P \leq 0.0009)$. Mist duration had a significant effect $(P \leq 0.025)$ on
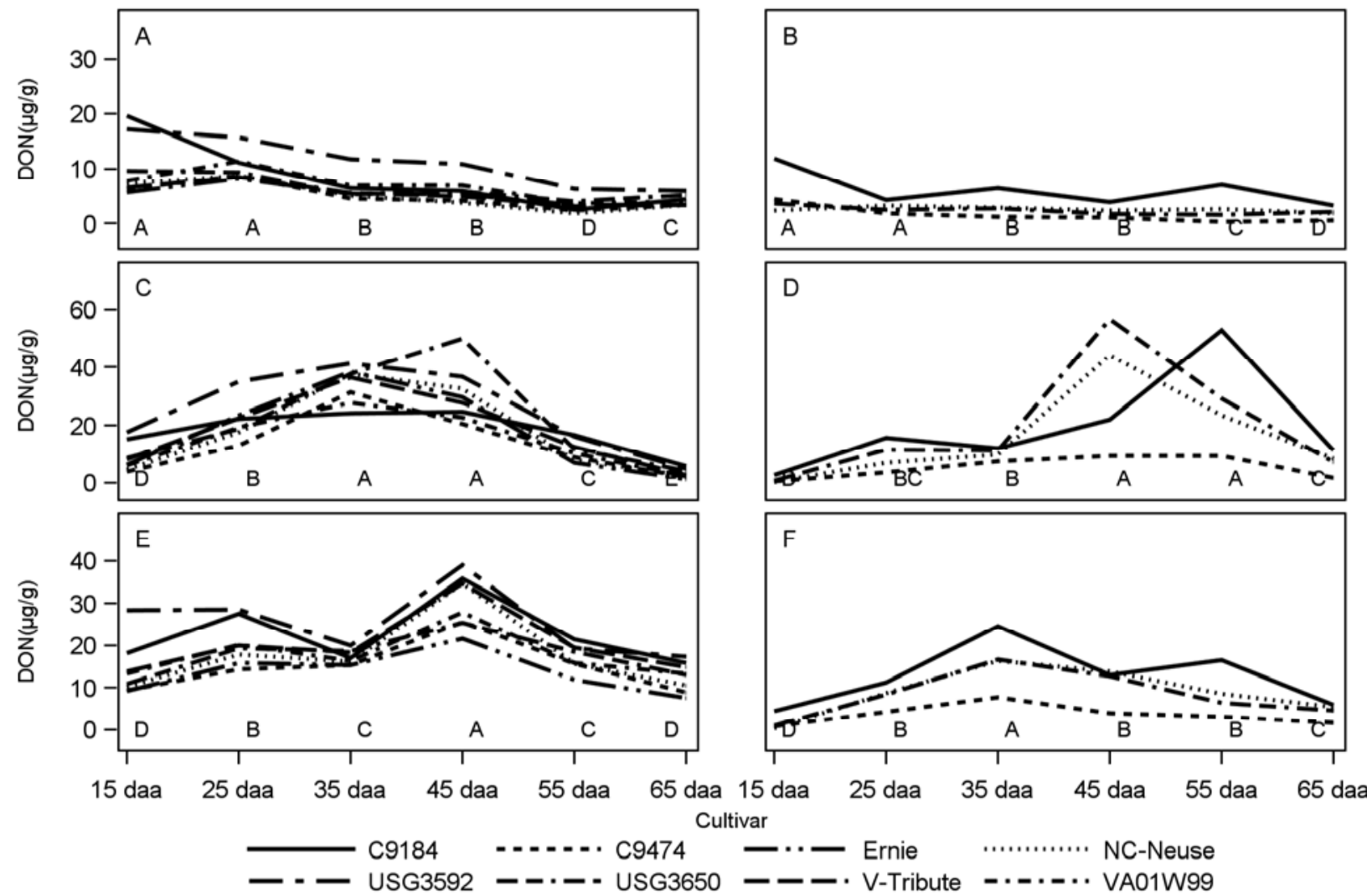

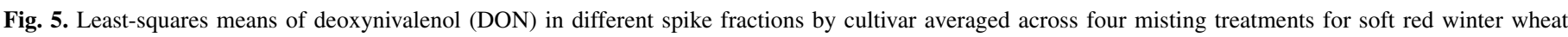

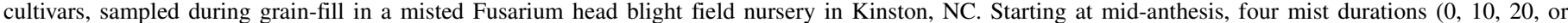

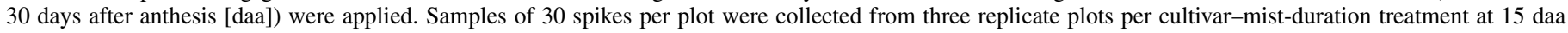

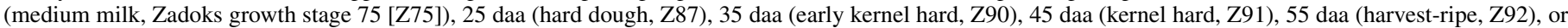

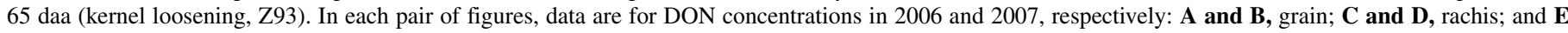

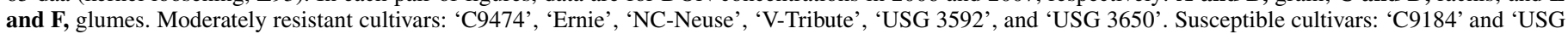
3592 '. Within a figure, means above the same letter are not significantly different $(P \leq 0.05$, Tukey's test $)$.

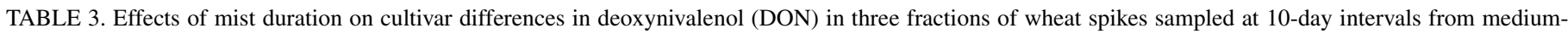
milk to kernel-loosening after inoculation at mid-anthesis with Fusarium graminearum macroconidia in a 2-year field experiment in Kinston, NC

\begin{tabular}{|c|c|c|c|c|c|c|c|c|c|}
\hline \multirow[b]{3}{*}{ Year, mist $(\mathrm{daa})^{\mathrm{y}}$} & \multicolumn{9}{|c|}{ Significance of cultivar effect on $\mathrm{DON}^{\mathrm{z}}$} \\
\hline & \multicolumn{3}{|c|}{ Grain } & \multicolumn{3}{|c|}{ Glumes } & \multicolumn{3}{|c|}{ Rachis } \\
\hline & $\mathrm{df}$ & $F$ value & $P$ value & $\mathrm{df}$ & $F$ value & $P$ value & $\mathrm{df}$ & $F$ value & $P$ value \\
\hline \multicolumn{10}{|l|}{2006} \\
\hline 0 & $7,52.7$ & 5.23 & 0.0001 & 7,16 & 21.35 & $<0.0001$ & $7,55.4$ & 6.31 & $<0.0001$ \\
\hline 10 & $7,57.3$ & 7.48 & $<0.0001$ & $7,14.6$ & 2.12 & 0.107 & $7,56.6$ & 2.31 & 0.039 \\
\hline 20 & $7,54.8$ & 1.43 & 0.213 & $7,13.7$ & 1.98 & 0.133 & $7,58.4$ & 2.07 & 0.061 \\
\hline 30 & $7,56.6$ & 1.35 & 0.245 & $7,14.2$ & 0.49 & 0.827 & $6,60.7$ & 1.76 & 0.122 \\
\hline \multicolumn{10}{|l|}{2007} \\
\hline 0 & $3,30.1$ & 17.18 & $<0.0001$ & $3,5.6$ & 19.04 & 0.002 & $3,36.1$ & 16.39 & $<0.0001$ \\
\hline 10 & $3,28.9$ & 13.52 & $<0.0001$ & $3,8.07$ & 14.81 & 0.001 & $3,36.2$ & 8.96 & 0.0001 \\
\hline 20 & $3,29.8$ & 12.29 & $<0.0001$ & $3,7.42$ & 2.96 & 0.103 & $3,35.5$ & 6.44 & 0.0001 \\
\hline 30 & $3,28.9$ & 5.47 & 0.004 & $3,6.74$ & 3.69 & 0.073 & $3,35.5$ & 6.82 & 0.0001 \\
\hline
\end{tabular}

y Mist duration in days after anthesis (daa). Data are from eight cultivars in 2006 and four of those eight in 2007.

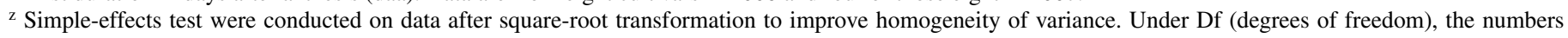
are numerator, denominator. 
grain DON in all samples except the 35-daa sample in 2006 and the 55-daa sample in 2007 ( $P=0.095$ and 0.076 , respectively; simple-effects test). Misting affected grain DON levels more so in 2006 than in 2007 (Fig. 4A and B). In the non-misted plots, mean grain DON was approximately the same in the 2 years across most sampling points, and post-anthesis mist tended to elevate DON levels.

Cultivar interacted significantly with sample in both years $(P \leq$ 0.013 ), and cultivars differed significantly for DON at all sample dates in both years (simple-effects test, $P \leq 0.006$ ). The susceptible C9184 had significantly higher DON $(P \leq 0.05$, data not shown) than the MR cultivars in the 15-daa sample in 2006 and in the 35-, 45-, and 55-daa samples in 2007; the susceptible USG 3592 had higher DON ( $P \leq 0.05$, data not shown) than all MR cultivars except USG 3650 and 'VA01W99' at all sample dates in 2006 (Fig. 5A and B).

Rachises. Mean DON concentrations were $20 \mu \mathrm{g} \mathrm{g}^{-1}$ in 2006 and $17 \mu \mathrm{g} \mathrm{g}^{-1}$ in 2007. DON reached similar maximal levels (30 to $50 \mu \mathrm{g} \mathrm{g}^{-1}$ ) in rachis tissues in both the wetter and the drier year (2006 and 2007, respectively) (Fig. 4C and D). Sample date interacted with both mist duration and cultivar in both years $(P \leq$ $0.0002)$. In 2006, the mist effect was significant in every sample (simple-effects test, $P \leq 0.002$ ), and peak rachis DON concentration lagged behind the end of misting by 15 to 25 days (Fig. 4C). In 2007, however, mist was only significant in the 35- and 55-daa samples (simple-effects test, $P \leq 0.002$; other samples, $P \geq 0.071$ ).

Cultivar was significant in all samples in $2007(P \leq 0.008)$ and in all but the 35- and 45-daa samples in 2006 ( $P \leq 0.005)$. Looking across the samples, cultivar FHB resistance rankings by severity (Table 1) were not as well reflected in rachis DON levels as they were in grain DON (Fig. 5). For example, rachis DON levels in the MR USG 3650 attained high peaks in both years. However, 'C9474', one of the most resistant cultivars, did have among the lowest rachis DON levels in both years, and the susceptible USG 3592 had significantly more DON than the MR cultivars at the first two sampling points in 2006 ( $P \leq 0.05$, data not shown). The susceptible C9184 did not attain the test-wide peak rachis DON concentration in 2006 but, in both years, it had high rachis DON concentrations at 55 daa.

In both years, mean rachis DON concentrations attained their peak at 45 daa (not different from 35 daa in 2006 and from 55 daa in $2007, P=0.05)$. Rachis DON levels were lowest $\left(5\right.$ to $\left.15 \mu \mathrm{g} \mathrm{g}^{-1}\right)$ at the earliest and latest sampling dates in both years. DON concentrations in rachises declined significantly from 45 daa to 65 daa.

Glumes. Sample date interacted significantly with mist duration $(P<0.0001)$ and cultivar $(P \leq 0.03)$ in 2006 but only with cultivar in $2007(P=0.01)$. Misting increased DON at all dates in 2006 $(P \leq 0.0007)$, except 25 and 65 daa (simple-effects test, $P=0.079$ and 0.126 , respectively). In 2007, misting affected DON only at 25 daa $(P=0.016)$. The magnitude of the difference in 2006 was greatest in the 45-daa sample, collected on 31 May, soon after a rain event of $20 \mathrm{~mm}$ on 27 May (Fig. 1).

Cultivars differed significantly for glume DON at all dates in both years (simple-effects test, $P \leq 0.021$ ), except at 35 and 45 daa in $2006(P \geq 0.164)$, the same two samples in which cultivars did not differ significantly for rachis DON that year. Glume DON levels of the susceptible USG 3592 were significantly higher than those of MR cultivars in the 15-daa sample $(P \leq 0.05$, data not shown), and levels of both susceptible cultivars (USG 3592 and C9184) remained higher than average throughout both years (Fig. 5E and F). In 2007, DON in C9184 glumes was higher than that in C9474 in all samples; the other cultivars were intermediate $(P \leq 0.05)$.

As with rachises, peak DON concentrations in glumes were found at the intermediate rather than the earliest and latest sampling dates (Fig. 4E and F). The samples with the highest mean DON were at 45 daa in 2006 and 35 daa in 2007. Mean glume
DON levels across samples, mist durations, and cultivars were significantly higher in 2006, the wetter year.

Whole-spike DON. In both years, estimated DON per spike was at its peak at 35 daa (Fig. 6). However, that peak was extended from 25 to 45 daa in 2006 . The mean proportions of total DON contributed by grain and glumes were roughly the same at almost all sample points. Due to their relatively low weight and volume, rachises contributed a small share of total spike DON despite their high DON concentrations, especially early and late in grain-fill.

Associations. Across sample dates, the most consistently significant associations were between DON in grain and DON in glumes, and between grain DON and FDK (Table 4). Severity, which was assessed shortly before the 25-daa samples were collected, was significantly associated with DON in grain and glumes of both 25- and 55-daa samples in 2006 (Table 4) $(P \leq 0.08)$. Severity was not associated with DON concentrations in rachises of either sample, however. In 2007, severity was not associated with DON in any spike fraction at either sample point.

The results of regression analysis to explore the relationship of grain DON at other sample dates with grain DON at 55 daa are shown in Table 5. There was a tendency for slopes to be more positive, and to differ significantly from zero, at 35 and 45 daa, compared with the earlier 15- and 25-daa stages.

\section{DISCUSSION}

This time-course study provides a new perspective on the progression of FHB symptoms during wheat spike development. Mean DON levels in wheat grain showed an overall declining trend during the period from medium milk ( 15 daa) to harvestripeness (55 daa). The decline is consistent with reports that DON biosynthesis occurs immediately following kernel infection, with DON acting as a virulence factor that allows fungal hyphae to move into the wheat rachis and spread to other florets (11). Initial kernel infections usually occur at and soon after anthesis, and the fungus then spreads through the head, infecting additional kernels over the following days. DON biosynthesis has been found (in a controlled environment) to be maximal at the infection front and before symptom development, and to drop after infection, shutting down by early dough (10), or $\approx 18$ daa. From the current study, it appears the conditions for DON biosynthesis may prevail longer in glume and rachis tissues, at least in the field.

Once synthesized, DON is thought to be removed by host detoxification (18) or by the leaching action of water (7). From the present results, it can be seen that, as Fusarium spreads from spikelet to spikelet via the rachis, maximum DON concentrations in the rachis are not attained until close to harvest. Maximum DON concentrations are also not reached in the glumes until close to harvest, although this dynamic appears to be more dependent on post-anthesis moisture. Thus, it appears unlikely that leaching due to rain or irrigation water is the reason for DON decline in grain, given the different time course of DON in the glumes. The late-season decline of DON concentrations in non-grain spike tissues may have causes similar to those in grain. In particular, conversion to glucosylated forms of DON could explain this decline, especially if the biosynthesis of DON in rachises and glumes starts later or lasts longer than that in grain.

At the same time that DON levels were dropping, FDK and PIK increased in the present experiment until after harvest ripeness in 2006 and until harvest ripeness in 2007. Misting increased early DON levels, FDK, and PIK and decreased sample weights to a greater extent in 2006 than in 2007. FDK and PIK at harvestripe were elevated in plots where moisture was applied after anthesis compared with unmisted plots. PIK and grain DON were not associated. Considering these data, it appears that Fusarium continues to spread within spikes through harvest ripeness without a concomitant increase in FDK or grain DON. Evidently, as spike tissues mature and dry down, the fungus continues its 
movement inside the head but is no longer able to colonize kernels as thoroughly as in earlier stages of grain fill. Moist conditions during grain-fill can alleviate the effects of increasing water shortage in the head, facilitating the ongoing process of kernel colonization needed for FDK. Without misting, FDK stayed relatively flat from early kernel-hard (35 daa) on, whereas PIK increased over time with or without misting. This suggests that less externally applied moisture is needed to allow fungal movement through the rachis for infection of additional kernels (PIK) than for colonization (FDK) and DON synthesis.

We found that, within wheat spikes, DON concentrations reached highest levels in the rachis; this is consistent with reports by researchers in New Zealand working with maize ears. Lauren and DiMenna (16) examined leaf and ear sections of maize infected with several Fusarium spp., including F. graminearum. They found that the most contaminated part of the ear was the rachis, with concentrations of DON plus nivalenol reaching 40 to $95 \mu \mathrm{g} \mathrm{g}^{-1}$, whereas the highest DON concentration in kernels was $3.8 \mu \mathrm{g} \mathrm{g}^{-1}$.

The results of this experiment confirm our earlier findings that increasing numbers of wet days in the post-anthesis period increase not only visible FHB symptoms but also kernel damage, the percentage of Fusarium-infected kernels, and DON levels in wheat spikes (6). Naturally, this relationship is substantiated for the volumes and durations of misting imposed; in this case, a maximum of 30 days at $\approx 10 \mathrm{~mm} \mathrm{day}^{-1}$. Longer durations or larger daily quantities of moisture could have a different effect.

What are the consequences of these findings for silage and forage producers? If FHB is observed or suspected, it is desirable to harvest whole plants on the early side (e.g., at milk rather than dough stage). The more conducive the year is to FHB, the closer to flowering one should aim to harvest.

The question arises whether variety ratings for FHB are useful in predicting whole-spike DON concentrations. Not surprisingly, differences in visible symptoms, which, along with grain DON concentrations, are the main variable used to compile cultivar FHB resistance rankings, were more associated with DON in grain than with DON in other spike tissues. Severity assessments at the normal time, $\approx 20$ days after mid-anthesis, were found to be associated with hard-dough levels of DON in grain and glumes in the higher-scab year but not in the lower-scab year. DON in grain was consistently associated with DON in glumes, and those are the two spike fractions contributing the majority of overall spike DON. On the whole, cultivar FHB resistance ratings should be useful in assessing which cultivars will have the highest levels of whole-plant DON when decisions must be made about harvest for silage. In other words, visible symptoms should have some relationship to whole-spike DON levels, especially in a bad scab year, when choices must be made whether to harvest whole crops for silage, or which fields or cultivars to ensile.

With respect to straw, it is clear that chaff can contain significant amounts of DON, as has been previously found. By weight, approximately half of total spike DON resides in glumes and rachises and, after the grain is harvested, that half remains in the chaff. Attention needs to be paid to the DON content of straw that may be consumed by animals. Averaged across mist-duration treatments, susceptible cultivars had higher DON concentrations in non-grain spike tissues than did MR cultivars at harvest. This has significance for use of wheat straw for bedding that might be eaten by livestock. It suggests that cultivar FHB ratings should be useful in choosing straw with minimal DON content. Straw harvested after prolonged post-anthesis rainy periods may have elevated concentrations of DON in the chaff. Although glume and rachis tissues would be a small proportion by mass of total straw, their high DON levels could be an issue in animal bedding or winter rations.

It would be useful to be able to predict DON levels in wheat at harvest time on the basis of DON levels measured earlier in the
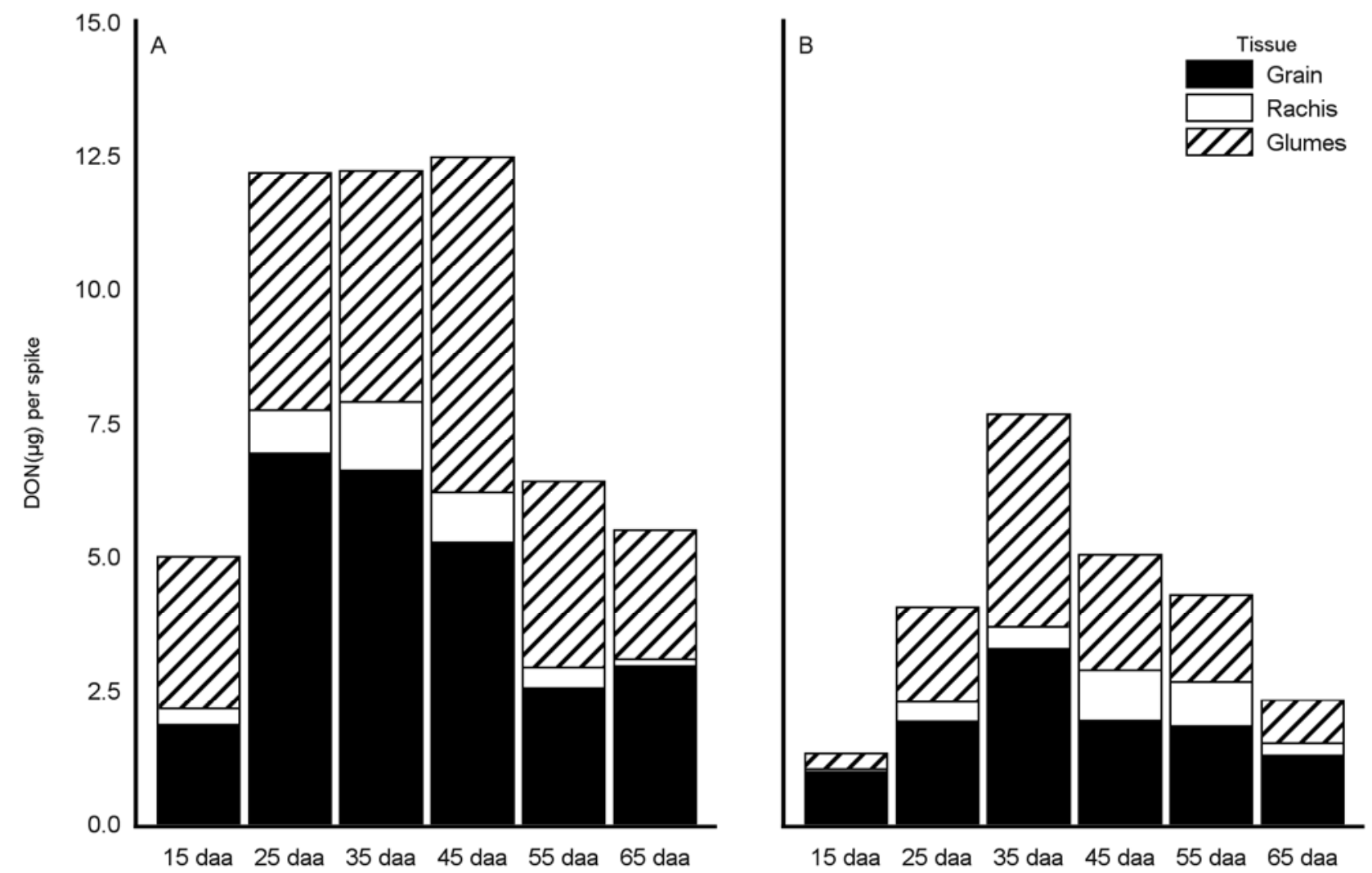

Fig. 6. Total deoxynivalenol (DON) per spike, based on spike fractions by weight and DON concentrations by spike fraction. Data averaged across A, eight soft red winter wheat cultivars in 2006 and B, four cultivars in 2007, subjected to four post-anthesis mist durations and sampled during grain-fill in a misted field nursery in Kinston, NC. 
grain-fill period. This would require that there be a demonstrable relationship between the two measures. Our results suggest that that relationship is affected by the number of moist days. The predictability of harvest-ripe DON by cultivar or in relationship to earlier DON concentrations tends to be diminished by increasing numbers of moist days in the post-anthesis period. Averaging across mist durations, it appears that DON is most predictive of harvest-ripe DON the closer in time that one samples to harvestripeness (i.e., sampling early in grain-fill may not provide an accurate estimate of grain DON concentrations at harvest). A model that includes visual symptoms and weather variables between anthesis and harvest, as well as early DON levels, might enhance the ability to predict DON at harvest.

Why did misting duration have less effect in 2007, the drier and later spring? It is possible that, in the drier year, amounts of mist were insufficient to affect disease development. Due to evaporation, applied mist may have been more subject to evaporation than in a wetter year and, thus, less available to affect disease development.

The mid-point of cultivar anthesis was used as the 0-daa mark although, in 2006, mid-anthesis varied by up to 6 days among cultivars. Single dates for turning off misting zones were unavoidable, given resource limitations. Although this method may have introduced some variation, if that variation were important, the downward-trending data in Figure 5A (for example) would exhibit more of a "traveling wave" pattern, with multiple, horizontally staggered lines based on inoculation date. No such pattern is apparent.

Severity levels were lower in plots destined to be misted for 30 days than in those misted for 20 days in 2007 (Table 1), and grain DON in the 30-day-misted plots was low at the first sampling date (Fig. 4B). The 30- and 20-day plots had received the same duration of mist at index assessment (i.e., the last 10 of the 30 days of mist had not yet been imposed). Nevertheless, factors in the 30-day block were initially unfavorable to disease development, in particular patches of WSBMV that retarded anthesis.

To summarize, the progression of DON differs among wheat spike tissues, with concentrations declining in grain during grainfill but peaking at approximately early kernel-hard in glumes and rachises. Fusarium continues spreading throughout the head during grain-fill but the ongoing production of FDK depends upon post-

TABLE 4. Selected associations of scab damage measures and deoxynivalenol (DON) in three fractions of wheat spikes sampled at 10-day intervals from mediummilk to kernel-loosening after inoculation at mid-anthesis with Fusarium graminearum macroconidia in a 2-year field experiment in Kinston, NC ${ }^{\mathrm{y}}$

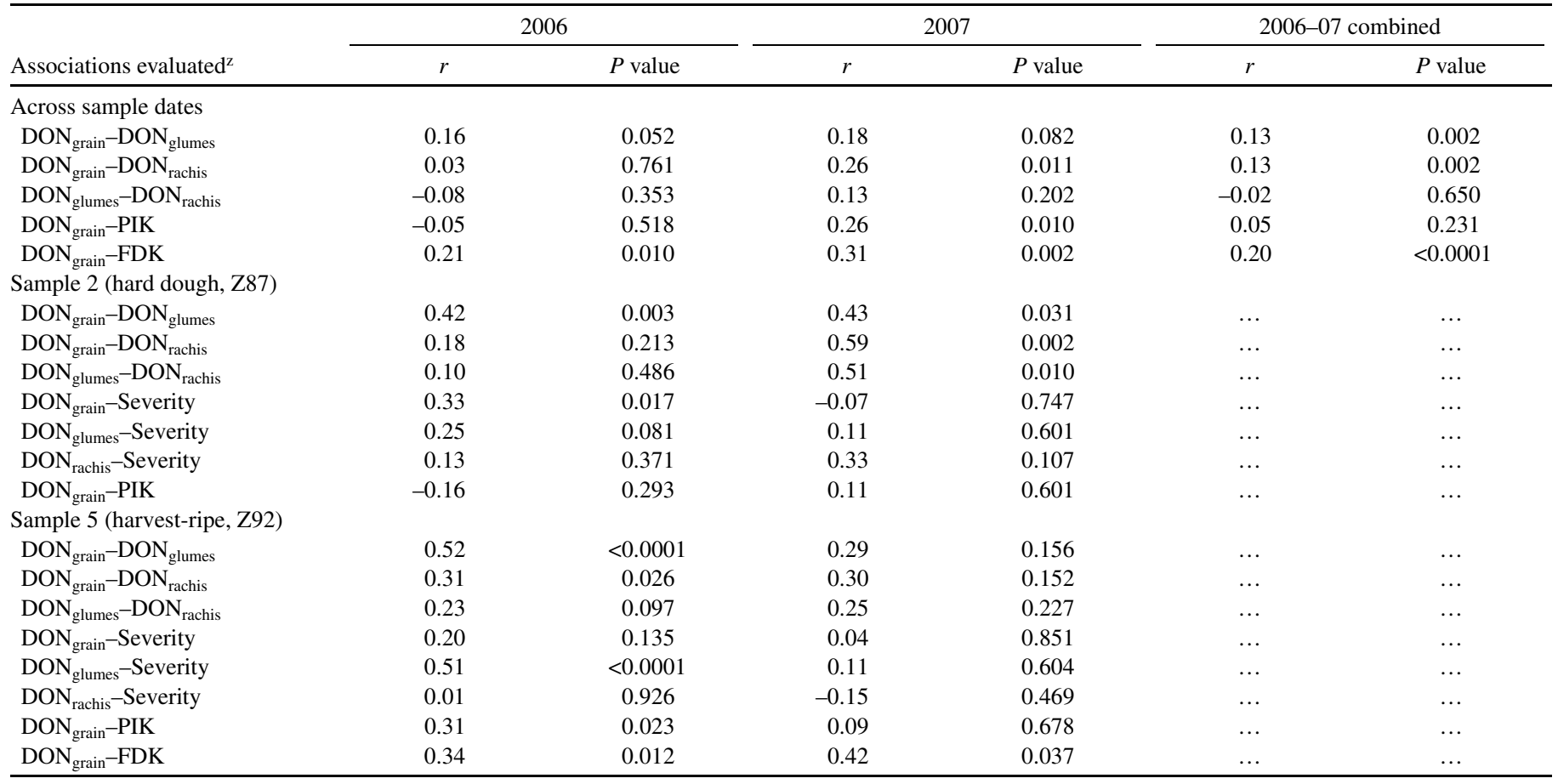

${ }^{\mathrm{y}}$ Data are from eight cultivars in 2006 and four of those eight in 2007, and were square-root transformed to improve homogeneity of variance. Correlations were assessed across cultivars and mist durations; $r=$ partial correlation coefficient.

${ }^{\mathrm{z}} \mathrm{PIK}=$ percent infected kernels; FDK = Fusarium-damaged kernels; severity was assessed at 20 days after anthesis (daa) or medium-dough stage. Sample dates were 15 daa (medium milk, Zadoks growth stage 75 [Z75]), 25 daa (hard dough, Z87), 35 daa (early kernel-hard, Z90), 45 daa (kernel hard, Z91), 55 daa (harvest-ripe, Z92), or 65 daa (kernel loosening, Z93). No data were available on PIK in 15-daa samples in 2007 and FDK in 15- and 25-daa samples in either year.

TABLE 5. Association of grain deoxynivalenol (DON) concentrations sampled at four points during grain-fill, with grain DON concentrations at harvest-ripe (sample 5, 55 days after anthesis [daa]) in eight soft red winter wheat cultivars grown in 2006 in Kinston, NCy

\begin{tabular}{|c|c|c|c|c|c|c|c|c|}
\hline \multirow[b]{2}{*}{ Mist $(\text { daa })^{\mathrm{z}}$} & \multicolumn{2}{|c|}{ Medium-milk (15 daa) } & \multicolumn{2}{|c|}{ Hard dough (25 daa) } & \multicolumn{2}{|c|}{ Early kernel-hard (35 daa) } & \multicolumn{2}{|c|}{ Kernel-hard (45 daa) } \\
\hline & Slope (s.e.) & $P$ value & Slope (s.e.) & $P$ value & Slope (s.e.) & $P$ value & Slope (s.e.) & $P$ value \\
\hline 0 & $-0.017(0.125)$ & 0.895 & $0.432(0.216)$ & 0.051 & $0.431(0.190)$ & 0.026 & $0.409(0.220)$ & 0.069 \\
\hline 10 & $0.397(0.119)$ & 0.001 & $0.138(0.123)$ & 0.264 & $0.364(0.108)$ & 0.001 & $0.420(0.057)$ & 0.010 \\
\hline 30 & $\ldots$ & $\ldots$ & $\ldots$ & $\ldots$ & $0.096(0.118)$ & 0.419 & $0.392(0.130)$ & 0.004 \\
\hline
\end{tabular}

${ }^{\mathrm{y}}$ DON concentrations were square-root transformed to improve homogeneity of variance. $P$ values are for the null hypothesis that the slope equals 0 ; s.e. $=$ standard error; ... indicates results not reported in cases where mist treatments had not yet been fully imposed.

${ }^{\mathrm{z}}$ Mist duration. 
anthesis moisture. Glumes and rachises account for approximately half the DON in wheat spikes, and DON from these sources must be taken seriously in small-grain straw that may be consumed by livestock. In bad FHB years, harvest of whole plants for silage should be conducted as soon as possible after flowering. Cultivar resistance ratings and observations of index at late-milk should be useful in making judgments about harvest of wholeplant silage and sourcing of straw.

\section{ACKNOWLEDGMENTS}

We thank the U.S. Wheat and Barley Scab Initiative for funding support; R. Parks, J. Patton-Özkurt, and the staff of Cunningham Research \& Extension Center for excellent technical assistance; and two anonymous reviewers for helpful comments which led to substantial improvements in the report.

\section{LITERATURE CITED}

1. Argyris, J., Van Sanford, D. A., and TeKrony, D. 2003. Fusarium graminearum infection during wheat seed development and its effect on seed quality. Crop Sci. 43:1782-1788.

2. Barneveld, R., and Edwards, A. 2004. Accounting for consumption of bedding material in deep litter housing systems-improving nutritional efficiency. Pork Ind. News 89:13-15.

3. Ben-Ghedalia, D., Kabala, A., Miron, J., and Yosef, E. 1995. Silage fermentation and in vitro degradation of monosaccharide constituents of wheat harvested at two stages of maturity. J. Agric. Food Chem. 43:24282431.

4. Bergen, W. G., Byrem, T. M., and Grant, A. L. 1991. Ensiling characteristics of whole-crop small grains harvested at milk and dough stages. J. Anim. Sci. 69:1766-1774.

5. Berthiller, F., Dall'Asta, C., Schuhmacher, R., Lemmens, M., Adam, G., and Krska, R. 2005. Masked mycotoxins: Determination of a deoxynivalenol glucoside in artificially and naturally contaminated wheat by liquid chromatography-tandem mass spectrometry. J. Agric. Food Chem. 53:3421-3425.

6. Cowger, C., Patton-Ozkurt, J., Brown-Guedira, G., and Perugini, L. 2009. Post-anthesis moisture increased Fusarium head blight and deoxynivalenol levels in North Carolina winter wheat field experiment. Phytopathology 99:320-327.

7. Culler, M. D., Miller-Garvin, J. E., and Dill-Macky, R. 2007. Effect of extended irrigation and host resistance on deoxynivalenol accumulation in Fusarium-infected wheat. Plant Dis. 91:1464-1472.

8. Galyean, M. L., and Defoor, P. J. 2003. Effects of roughage source and level on intake by feedlot cattle. J. Anim. Sci. 81:E8-16.

9. Gillespie, J. R., and Flanders, F. B. 2010. Modern Livestock and Poultry Production. Delmar Cengage Learning, Clifton Park, NY.

10. Hallen-Adams, H. E., Wenner, N., Kuldau, G. A., and Trail, F. 2011. Deoxynivalenol biosynthesis-related gene expression during wheat kernel colonization by Fusarium graminearum. Phytopathology 101:1091-1096.

11. Jansen, C., von Wettstein, D., Wilhelm, S., Kogel, K.-H., Felk, A., and Maier, F. J. 2005. Infection patterns in barley and wheat spikes inoculated with wild-type and trichodiene synthase gene disrupted Fusarium graminearum. Proc. Natl. Acad. Sci. USA 102:16892-16897.

12. Juskiw, P. E., Helm, J. H., and Salmon, D. F. 2000. Forage yield and quality for monocrops and mixtures of small grain cereals. Crop Sci. 40:138-147.

13. Keady, T. W. J. 2005. Ensiled maize and whole crop wheat forages for beef and dairy cattle: effects on animal performance. Pages 65-82 in: Silage Production and Utilisation. R. S. Park and M. D. Stronge, eds. Wageningen Academic Publishers, Wageningen, The Netherlands.

14. Khorasani, G. R., Jedel, P. E., Helm, J. H., and Kennelly, J. J. 1997. Influence of stage of maturity on yield components and chemical composition of cereal grain silages. Can. J. Anim. Sci. 77:259-267.

15. Komada, H. 1976. A new selective medium for isolating Fusarium from natural soil. Proc. Am. Phytopathol. Soc. 3:221.

16. Lauren, D. R., and DiMenna, M. E. 1999. Fusaria and Fusarium mycotoxins in leaves and ears of maize plants 2 . A time course study made in the Waikato region, New Zealand, in 1997. N. Z. J. Crop Hortic. Sci. 27:215-223.

17. Lemmens, M., Buerstmayr, H., Krska, R., Schuhmacher, R., Grausgruber, H., and Ruckenbauer, P. 2004. The effect of inoculation treatment and long-term application of moisture on Fusarium head blight symptoms and deoxynivalenol contamination in wheat grains. Eur. J. Plant Pathol. 110:299-308.

18. Lemmens, M., Scholz, U., Berthiller, F., Dall'Asta, C., Koutnik, A.,
Schuhmacher, R., Adam, G., Buerstmayr, H., Mesterhazy, A., Krska, R., and Ruckenbauer, P. 2005. The ability to detoxify the mycotoxin deoxynivalenol colocalizes with a major quantitative trait locus for Fusarium head blight resistance in wheat. Mol. Plant-Microbe Interact. 18:13181324.

19. Littell, R. C., Milliken, G. A., Stroup, W. W., Wolfinger, R. D., and Schabenberber, O. 2006. SAS for Mixed Models, 2nd ed. SAS Institute, Inc., Cary, NC.

20. Matthäus, K., Dänicke, S., Vahjen, W., Simon, O., Wang, J., Valenta, H., Meyer, K., Strumpf, A., Ziesenib, H., and Flachowsky, G. 2004. Progression of mycotoxin and nutrient concentrations in wheat after inoculation with Fusarium culmorum. Arch. Anim. Nutr. 58:19-35.

21. McCartney, D. H., Block, H. C., Dubeski, P. L., and Ohama, A. J. 2006. Review: The composition and availability of straw and chaff from small grain cereals for beef cattle in western Canada. Can. J. Anim. Sci. 86:443455 .

22. McMullen, M., Jones, R., and Gallenberg, D. 1997. Scab of wheat and barley: A re-emerging disease of devastating impact. Plant Dis. 81:1340-1348.

23. Mesterhazy, A. 1995. Types and components of resistance to Fusarium head blight of wheat. Plant Breed. 114:377-386.

24. Miller, J. D., and Young, J. C. 1985. Deoxynivalenol in an experimental Fusarium graminearum infection of wheat. Can. J. Plant Pathol. 7:132-134.

25. Miller, J. D., Young, J. C., and Sampson, D. R. 1985. Deoxynivalenol and Fusarium head blight resistance in spring cereals. Phytopathol. Z. 113:359-367.

26. Moore, D. D. 2005. Occurrence of mycotoxins in straw used in pig deep litter systems in Australia. Pages 102 in: Proc. Annu. Conf. Br. Soc. Anim. Sci. York, UK.

27. Murphy, J. P., and Navarro, R. 2001. Pages 1-19 in: Uniform Southern Soft Red Winter Wheat Fusarium Head Blight Screening Nursery, 2001 Nursery Report. North Carolina State University, Raleigh.

28. Murphy, J. P., Navarro, R., and Srnic, G. 2000. Pages 1-14 in: Uniform Southern Soft Red Winter Wheat Fusarium Head Blight Screening Nursery, 2000 Nursery Report. North Carolina State University, Raleigh.

29. Murphy, J. P., Navarro, R. A., and Van Sanford, D. A. 2002. Pages 1-23 in: Uniform Southern Soft Red Winter Wheat Fusarium Head Blight Screening Nursery, 2002 Nursery Report. North Carolina State University, Raleigh.

30. Murphy, J. P., Navarro, R. A., and Van Sanford, D. A. 2003. Pages 1-28 in: Uniform Southern Soft Red Winter Wheat Fusarium Head Blight Screening Nursery, 2003 Nursery Report. North Carolina State University, Raleigh.

31. Paul, P. A., Lipps, P. E., and Madden, L. V. 2005. Relationship between visual estimates of Fusarium head blight intensity and deoxynivalenol accumulation in harvested wheat grain: a meta-analysis. Phytopathology 95:1225-1236

32. Rottinghaus, G. E., Tacke, B. K., Evans, T. J., Mostrom, M. S., Sweets, L. E., and McKendry, A. L. 2009. Fusarium mycotoxin concentrations in the straw, chaff, and grain of soft red winter wheats expressing a range of resistance to Fusarium head blight. Page 10 in: National Fusarium Head Blight Forum. S. M. Canty, A. Clark, J. Mundell, E. Walton, D. Ellis, and D. A. Van Sanford, eds. University of Kentucky, Orlando, FL.

33. Sasanya, J. J., Hall, C., and Wolf-Hall, C. 2008. Analysis of deoxynivalenol, masked deoxynivalenol, and Fusarium graminearum pigment in wheat samples, using liquid chromatography-UV-mass spectrometry. J. Food Prot. 71:1205-1213.

34. Saxton, A. M. 1998. A macro for converting mean separation output to letter groupings in Proc Mixed. Pages 1243-1246 in: 23rd SAS Users Group International. SAS Institute, Cary, NC.

35. Scott, P. M., Nelson, K., Kanhere, S. R., Karpinski, K. F., Hayward, S., Neish, G. A., and Teich, A. H. 1984. Decline in deoxynivalenol (vomitoxin) concentrations in 1983 Ontario winter wheat before harvest. Appl. Environ. Microbiol. 48:884-886.

36. Staples, C. R. 2003. Harvesting small grain crops for silage. Dairy Science Department, University of Florida, Gainesville, FL. http:// edis.ifas.ufl.edu/pdffiles/DS/DS15500.pdf

37. Sutton, J. D., Phipps, R. H., Deaville, E. R., Jones, A. K., and Humphries, D. J. 2002. Whole-crop wheat for dairy cows: effects of crop maturity, a silage inoculant and an enzyme added before feeding on food intake and digestibility and milk production. Anim. Sci. 74:307-318.

38. Teich, A. H. 1989. Epidemiology of wheat (Triticum aestivum L.) scab caused by Fusarium spp. Pages 269-282 in: Fusarium: Mycotoxins, Taxonomy and Pathogenicity. J. Chelkowski, ed. Elsevier, Amsterdam.

39. Valderas-Gomez, E., Schaalje, G. B., and Fellingham, G. W. 2007. Performance of the Kenward-Roger method when the covariance structure is selected using AIC and BIC. Commun. Stat. Simul. Comput. 34:377-392.

40. Waldron, B. L., Moreno-Sevilla, B., Anderson, J. A., Stack, R. W., and Frohberg, R. C. 1999. RFLP mapping of QTL for Fusarium head blight resistance in wheat. Crop Sci. 39:805-811.

41. Wilkinson, J. M. 1999. Silage and animal health. Nat. Toxins 7:221-232. 